\title{
Is Multidrug Resistance in Acute Otitis Media with Streptococcus pneumoniae Associated with a More Severe Disease?
}

\author{
Raluca Horhat $^{a, b}, f$ Florin-Raul Horhat ${ }^{c}$ Valeria Mocanu ${ }^{d, e}$ \\ aDepartment of Functional Sciences, Biophysics, "Victor Babeș" University of Medicine and Pharmacy Timișoara, \\ Timisoara, Romania; ${ }^{b}$ Clinic of Paediatric Surgery (Otolaryngology Department), Emergency Children's Hospital \\ Louis Turcanu, Timisoara, Romania; 'Department of Functional Sciences, Medical Informatics and Biostatistics, \\ "Victor Babeș" University of Medicine and Pharmacy, Timisoara, Romania; 'Department of Surgery, Ophthalmology,

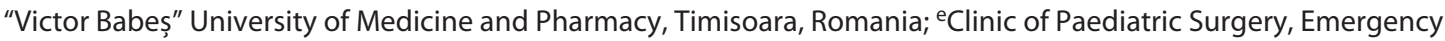 \\ Children's Hospital Louis Turcanu, Timisoara, Romania; ${ }^{f}$ Center for Modeling Biological Systems and Data Analysis, \\ "Victor Babeş" University of Medicine and Pharmacy, Timisoara, Romania
}

\section{Highlights of the Study}

- Multidrug-resistant (MDR) S. pneumoniae was associated with higher values of inflammatory markers in severe acute otitis media (AOM).

- C-reactive protein had the highest predictive value for the presence of MDR S. pneumoniae in the first $48 \mathrm{~h}$ of disease evolution.

- Absolute neutrophil count and percentage of neutrophils showed limited practical applicability.

- AOM with MDR S. pneumoniae presented different spectra of associated pathology and/or complications.

\section{Keywords}

Multidrug-resistant Streptococcus pneumoniae .

Inflammatory response $\cdot$ C-reactive protein $\cdot$ Neutrophils .

Otorrhea - Mastoiditis

\section{Abstract \\ Objectives: Streptococcus pneumoniae is the leading bacte- rial etiologic agent in acute otitis media ( $A O M)$, and it pro- duces a more severe inflammatory response than other oto- pathogens. Additionally, the presence of multidrug-resis- tant (MDR) S. pneumoniae is an important issue in the management of AOM. The present pilot study aimed to as-}

karger@karger.com www.karger.com/mpp

Karger $\stackrel{\text { ' }}{5}$
(C) 2021 The Author(s)

Published by S. Karger AG, Basel

This is an Open Access article licensed under the Creative Commons Attribution-NonCommercial-4.0 International License (CC BY-NC) (http://www.karger.com/Services/OpenAccessLicense), applicable to the online version of the article only. Usage and distribution for commercial purposes requires written permission. certain whether MDR S. pneumoniae is associated with a higher inflammatory response and/or a more severe disease. Methods: This was a prospective, single-center study on nonpneumococcal conjugate vaccine-immunized pediatric patients with severe AOM. Demographic and clinical characteristics were recorded. Middle ear fluid was obtained and cultured for each patient; antibiotic-resistance profiling was tested for S. pneumoniae isolates. The C-reactive protein (CRP) level and complete blood count were determined. Patients with positive middle ear fluid culture for S. pneumoniae were divided into 2 groups according to antibiotic resistance profile: MDR and non-MDR. Results: MDR S. pneumoniae was identified in 15 (35.7\%) of the 42 eligible patients. 
Children in this group had significantly higher CRP levels $(72.23 \pm 62.92$ vs. $14.96 \pm 15.57 \mathrm{mg} / \mathrm{L}, p<0.001)$, higher absolute neutrophil count $\left(8.46 \pm 3.97\right.$ vs. $5.22 \pm 4.5 \times 10^{3} / \mathrm{mm}^{3}$, $p=0.004)$, higher percentage of neutrophils $(52.85 \pm 13.49 \%$ vs. $38.34 \pm 16.16 \%, p=0.004)$, and were more prone to develop acute mastoiditis $(p=0.01)$. Receiver operating characteristic analysis identified CRP as the best biomarker to discriminate between the 2 groups of patients (AUC $=0.891$ ). Conclusion: MDR S. pneumoniae was associated with a more severe inflammatory response and a higher incidence of mastoiditis.

(C) 2021 The Author(s)

Published by S. Karger AG, Basel

\section{Introduction}

Acute otitis media (AOM) is a common pediatric infectious disease and the leading bacterial infection of childhood. By the age of 5 years, $80 \%$ of children present at least one episode, with $30-40 \%$ of these experiencing recurrent episodes $[1,2]$.

Although most AOM infections are of viral origin, coinfection with bacteria is encountered in $65 \%$ of cases, and the most frequently reported pathogen is Streptococcus pneumoniae (S. pneumoniae) [3]. It is a human-specific pathogen colonizing the nasopharynx. Higher rates of carriage are associated with infancy, cold season, and childcare [2]. S. pneumoniae infection is of interest as not only is it frequently associated with more severe symptoms, mastoiditis, as well as with pneumonia and invasive pneumococcal disease [4] but also with an increased inflammatory response to the infection [5]. The wide spectrum of disease produced by the organism is due to virulence factors such as the capsule, toxins, surface-associated proteins, and genome variation [2]. Regarding the inflammatory response, previous studies have shown that white blood cells and absolute neutrophil count (ANC) values are higher for AOM caused by S. pneumoniae than those of other etiological agents [5]. Furthermore, $S$. pneumoniae is the most prevalent pathogen among patients with AOM who experience treatment failure [6].

Irrespective of the etiology of AOM in pediatric patients, current treatment guidelines by the American Academy of Pediatrics [7] strongly recommend that young children 6 months and older be treated with antibiotics on an empirical basis if the disease is clinically severe. First-line treatment includes high-dose amoxicillin or an antibiotic with additional $\beta$-lactamase coverage (amoxicillin-clavulanate or ceftriaxone) [7]. Therefore, AOM is a major reason for antibiotic prescription in pediatric patients and consequently the presence of antibiotic resistance is an important issue in AOM management. Resistance to 3 or more classes of antibiotics classifies the strain of a bacterial pathogen as multidrug-resistant (MDR).

In some geographic locations, MDR S. pneumoniae is commonly resistant to both of the recommended initial treatments and is one of the main causes for treatment failure [8] and a problem of major concern for public health $[9,10]$. To overcome the effect of antibiotic resistance, tympanocentesis and culture of middle ear fluid are necessary. However this is not routinely performed due to possible complications, and thus, the identification of the appropriate antibiotic treatment is often delayed. The aims of the present study were to investigate the clinical features of severe AOM caused by MDR S. pneumoni$a e$ and to examine the relationship between common inflammatory markers and the presence of multidrug resistance (MDR).

\section{Subjects and Methods}

\section{Design}

This was a pilot, single-center, prospective study of hospitalized pediatric patients diagnosed with severe acute otitis media due to $S$. pneumoniae. All patients provided clinical and biomarker samples and were treated according to the same standard of care. Upon completion of patient enrollment and study participation, the biomarker data derived from each patient were grouped by the MDR status of the patient's pathogen, and the predictive nature of each biomarker assessed, as shown in Figure 1. The data that support the findings of this study are available in the "figshare" repository at https://figshare.com/articles/software/AOM/ 12959822 .

\section{Participants}

Pediatric patients aged between 6 months and 5 years were eligible for the study and were required to meet all of the following inclusion criteria: (1) acute onset of AOM symptoms (fever, irritability, tugging of the ear, or earache), lasting for $<48 \mathrm{~h}$; (2) physical findings consistent for AOM (bulging of tympanic membrane and blurring of its anatomical landmarks or pus drainage from the ear) lasting <24 h; (3) symptoms suggesting severe AOM [7]: high fever $\left(>39^{\circ} \mathrm{C}\right)$ and/or moderate/severe otalgia; (4) no antibiotic treatment in the 10 days before presentation at hospital.

Patients were excluded from the study if any of the following were found: (1) presence of tympanostomy tubes; (2) patient had received pneumococcal conjugate vaccine immunization; (3) known immunodeficiency; and (4) known recurrent or refractory otitis media. Patients were admitted between October 1, 2017, and September 31, 2019, in the Emergency Children's Hospital Louis Turcanu, Timisoara, Romania. A 2-step enrollment was performed. Patients were selected initially based on the above mentioned clinical criteria. A second selection was performed based on the result of the culture from the middle ear fluid (MEF), selecting only the patients with $S$. pneumoniae as the etiological agent as shown in Figure 1. 


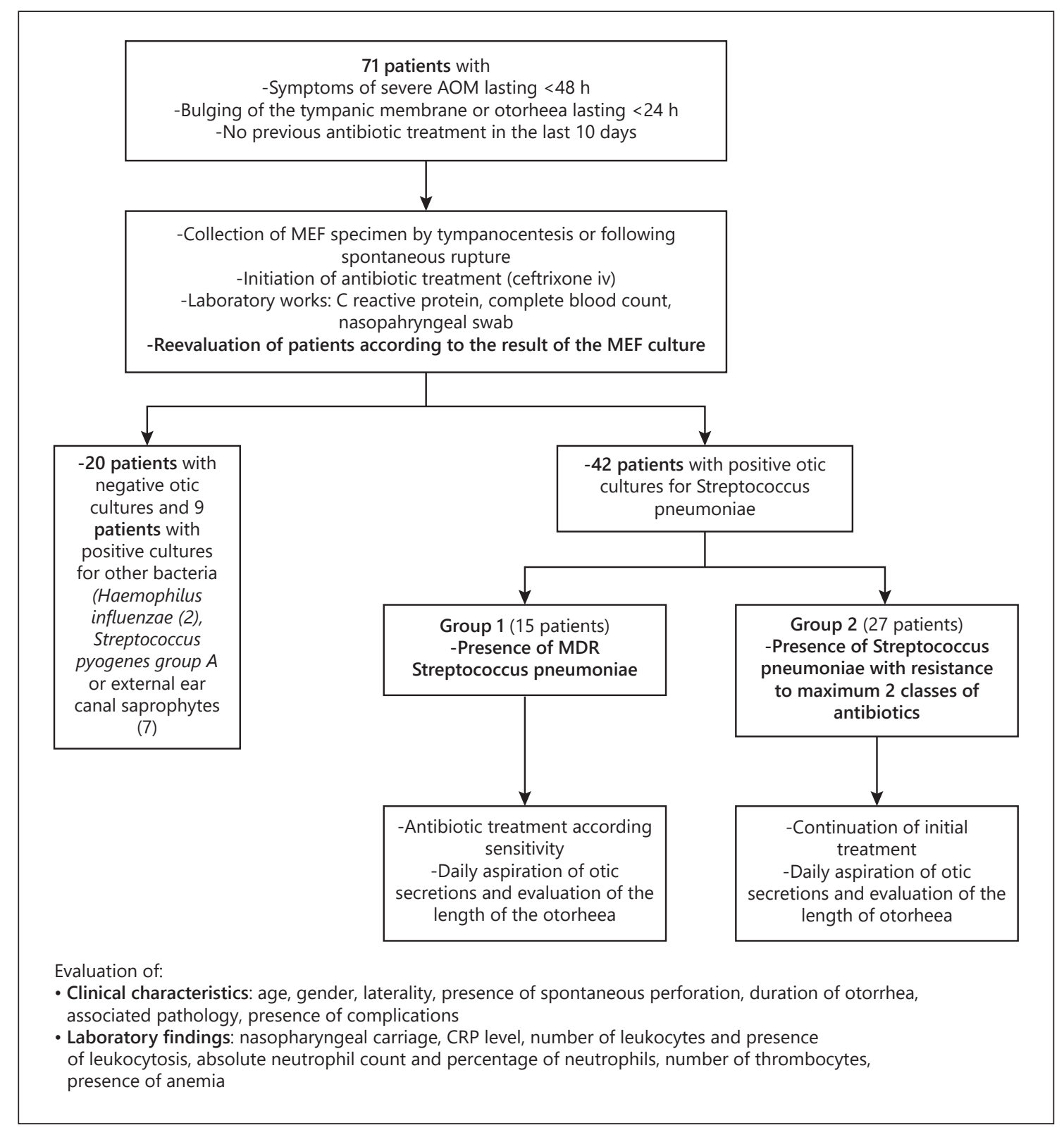

Fig. 1. Study flowchart and disposition of patients. AOM, acute otitis media; CRP, C-reactive protein; MEF, middle ear fluid; MDR, multidrug resistant.

\section{Methods}

Every child underwent a complete otolaryngological examination, and when myringotomy was required, it was performed in the anterior-inferior quadrant. Prior to tympanocentesis, the external ear canal was rinsed with an antiseptic solution (povidone/iodine), and middle ear fluid was collected using a designated sterile cotton swab (Meus Srl, Piove di Sacco, Italy) from very near to the myringotomy site. In children with spontaneous perforation, fluid was obtained in a similar manner, after aspiration of the secretions in the external ear canal. All samples were sent to the microbiology laboratory in Amies transport medium (Meus Srl, Piove di Sacco, Italy) and processed within $8 \mathrm{~h}$. Isolates were identified using stan- dard microbiological methods: Gram stain morphology, optochin and bile salt tests, antigenic testing, and biochemical methods. Antimicrobial susceptibility of $S$. pneumoniae was evaluated using a minimum inhibitory concentration method using Vitek 2 (bioMerieux) [11] and was defined according to Clinical and Laboratory Antimicrobial Standards Institute 2017 criteria [12]. Intermediate or full resistance was defined as nonsusceptibility. Resistance to $>1$ beta-lactam antibiotic (penicillin, amoxicillin, cefotaxime, ceftriaxone, or imipenem) was considered resistance to beta-lactams. Resistance to 3 or more antibiotic classes was defined as MDR.

Nasal and pharyngeal swabs, blood cell count, and C-reactive protein (CRP) analysis were part of the routine clinical care. Nasal 
Table 1. Antimicrobial susceptibility of S. pneumoniae isolates causing severe acute otitis media

\begin{tabular}{|c|c|c|c|c|}
\hline \multirow[t]{2}{*}{ Antimicrobial agents } & \multirow{2}{*}{$\begin{array}{l}\text { Susceptible-MIC } \\
\text { breakpoint, } \mu \mathrm{g} / \mathrm{mL}\end{array}$} & \multicolumn{3}{|l|}{ Isolates, $n(\%)$} \\
\hline & & $\begin{array}{l}\text { Group } 1(n=15) \\
\text { isolates, } n\end{array}$ & $\begin{array}{l}\text { Group } 2(n=27) \\
\text { isolates, } n\end{array}$ & $\begin{array}{l}\text { total }(n=42) \\
\text { isolates, } n\end{array}$ \\
\hline Penicillin & $\leq 2$ & $2(13.33)$ & $24(8.89)$ & 26 (61.9) \\
\hline Amoxicillin/clavulanate & $\leq 2 / 1$ & $4(26.67)$ & $27(100)$ & $31(73.81)$ \\
\hline Cefotaxime & $\leq 1$ & 7 (46.67) & $27(100)$ & $34(80.95)$ \\
\hline Ceftriaxone & $\leq 1$ & $8(53.33)$ & $27(100)$ & $35(83.33)$ \\
\hline Erythromycin & $\leq 0.25$ & $0(0)$ & $9(33.33)$ & $9(21.43)$ \\
\hline Chloramphenicol & $\leq 4$ & $15(100)$ & $27(100)$ & $42(100)$ \\
\hline Tetracycline & $\leq 1$ & $3(20)$ & $22(81.48)$ & $25(59.52)$ \\
\hline Levofloxacin & $\leq 2$ & $15(100)$ & $27(100)$ & $42(100)$ \\
\hline TMP/SMX & $\leq 0.5 / 9.5$ & $0(0)$ & $7(25.93)$ & $7(16.67)$ \\
\hline Vancomycin & $\leq 1$ & $15(100)$ & $27(100)$ & $42(100)$ \\
\hline
\end{tabular}

Isolates with intermediate or full resistance were defined as nonsusceptible. TMP/SMX, trimethoprim/ sulfamethoxazole; MIC, minimal inhibitory concentration; S. pneumoniae, Streptococcus pneumoniae.

and pharyngeal swabs were obtained from all study participants and considered positive if $S$. pneumoniae was present with the same antibiotic resistance pattern as in the MEF culture. Blood samples for inflammatory tests were collected at admission. CRP was measured using routine analysis methodology (turbidimetric method, Cobas Integra 400 Plus) [13]. Complete blood count parameters were obtained by fluorescence flow cytometry on a Sysmex XT 4000i automated hematology analyzer [14]. Blood cultures were conducted when considered necessary, in order to diagnose concurrent pneumococcal bacteremia. If lower respiratory tract symptomatology was present, chest X-rays were performed.

Otoscopic examination was performed daily, with clinical evaluation and aspiration of middle ear secretions. Otorrhea was evaluated as negative if absent for 2 consecutive days. Its duration was considered from tympanotomy/spontaneous perforation until the first day of absence.

Antibiotic treatment for all study participants was initiated with intravenous ceftriaxone $(50 \mathrm{mg} / \mathrm{kg})$ and continued according to the antibiotic susceptibility when the results were available. Intravenous treatment was continued throughout the hospitalization period. After clinical remission and resolution of otorrhea, patients were discharged, and if necessary, oral antibiotic treatment was prescribed. The total duration of the antibiotic treatment was variable, depending on the evolution of the disease.

\section{Statistics}

Statistical analysis was performed using $\mathrm{R}$ software version 3.6.2 [15]. The data derived from eligible patients were divided into 2 groups according to bacterial antibiotic resistance. The analyzed variables were divided in 2 categories: clinical characteristics and laboratory findings, as illustrated in Figure 1.

Means and standard deviations were computed. Normality of data was checked by the Shapiro-Wilk test. The statistical difference between the 2 study groups was tested using an unpaired $t$ test for continuous variables if the normal distribution was present, otherwise the Mann-Whitney U test was employed. For categorical data, contingency tables and the $\chi^{2}$ and/or Fischer test were used.
In order to analyze the markers of inflammation between the 2 groups, we calculated the area under the receiver operating characteristic (ROC) curves (AUC). The best biomarker threshold was evaluated using both Youden's J statistic and the closest top-left technique. The duration of otorrhea was analyzed by Kaplan-Meier curves. $p$ values $<0.05$ were considered significant. The minimum sample size estimated for an $\mathrm{AUC}=0.75$, power $=0.8$ is 12 cases and 23 controls.

\section{Results}

Based on the clinical criteria, 71 patients were initially enrolled (Fig. 1). Out of these 71 patients, 29 did not provide a positive culture for S. pneumoniae as shown in Figure 1. The 42 remaining patients were divided into 2 groups according to antibacterial resistance (Table 1); Group 1 consisted of 15 patients with MDR S. pneumoniae and Group 2 consisted of 27 patients with non-MDR S. pneumoniae.

\section{Clinical Characteristics}

There were more male patients in the MDR pneumococcus group $(p=0.02)$ (Table 2$)$. The associated pathology was broadly similar between the groups, with the exception of pneumonia, for which there was a higher incidence (at the limit of statistical significance) in patients with non-MDR $S$. pneumoniae $(p=0.05)$. Regarding complications arising from AOM, mastoiditis was seen only in the MDR group ( 4 of 15 patients, $p=0.01$ ). Although it did not reach statistical significance, concurrent pneumococcal bacteremia was more frequently seen in 
Table 2. Clinical and epidemiological characteristics for the patients in the 2 study groups (Group 1- otitis media with MDR S. pneumoniae, Group 2 - otitis media with non-MDR S. pneumoniae)

\begin{tabular}{llll}
\hline Variable & Group 1 $(n=15)$ & Group 2 $(n=27)$ & $p$ value \\
\hline Age mean \pm SD (range), months & $23.07 \pm 12.29[6-46]$ & $19.89 \pm 10.77[6-42]$ & 0.41 \\
Age $<12$ months (yes:no) & $5: 10$ & $10: 17$ & 0.73 \\
Age $<24$ months (yes:no) & $9: 6$ & $17: 10$ & 1 \\
Gender (male:female) & $13: 2$ & $12: 15$ & 0.009 \\
Laterality (unilateral:bilateral) & $2: 13$ & $11: 16$ & 0.3 \\
Spontaneous perforation & 0 & 5 & 0.14 \\
Length of otorrhea mean $\pm S D$ (range), days & $5.4 \pm 1.91[2-9]$ & $3 \pm 1.3[1-6]$ & $<0.001$ \\
Associated pathology & 1 & 2 & 1 \\
$\quad$ Bronchiolitis & 2 & 12 & 0.049 \\
$\quad$ Pneumonia & 7 & 16 & 0.42 \\
$\quad$ Anemia & 0 & 2 & 0.52 \\
$\quad$ Asthma & 4 & 0 & 0.01 \\
Complications & & 1 & 0.28 \\
$\quad$ Mastoiditis & 2 & & \\
\hline
\end{tabular}

SD, standard deviation; S. pneumoniae, Streptococcus pneumoniae.

Fig. 2. Kaplan-Meier curves for the length

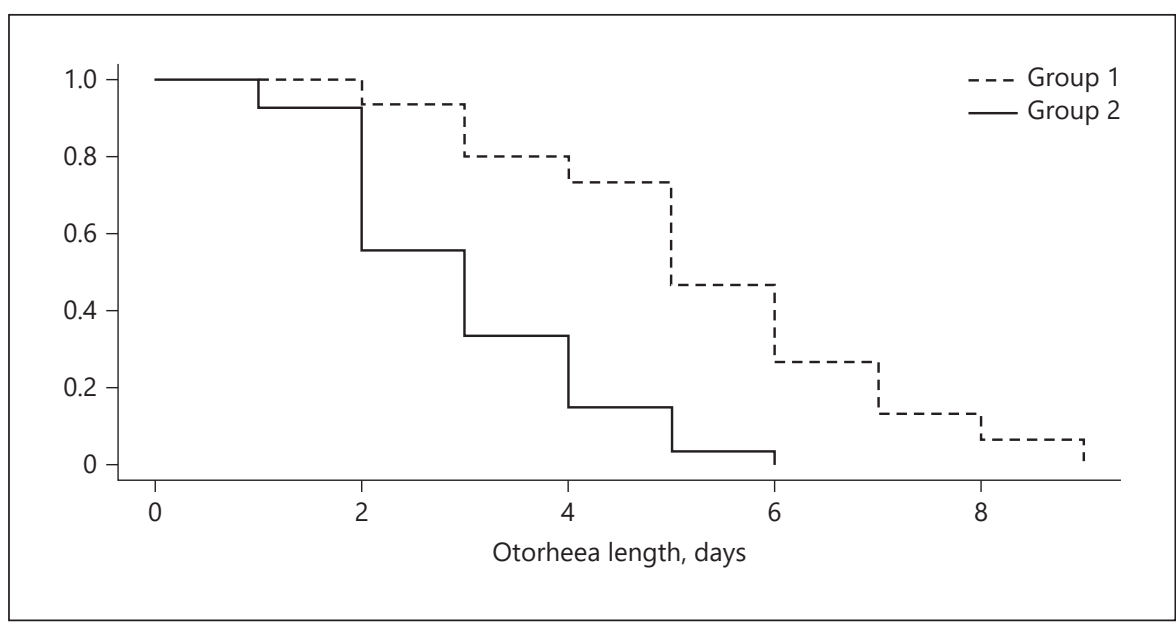

of the otorrhea in the 2 groups.

the MDR group (13.33\% vs. 3.7\%). Regarding otorrhea, there was a statistical difference $(p<0.001)$ between the 2 groups with the MDR patients recovering more slowly $(5.4 \pm 1.91$ days) than the patients in the non-MDR group ( $3 \pm 1.3$ days) (Table 2; Fig. 2). There were no adverse events associated with tympanocentesis.

\section{Laboratory Findings}

The results for the laboratory findings are shown in Table 3. Nasopharyngeal carriage was detected in 18 of the patients $(42.86 \%)$, with no statistical significant difference between the 2 study groups ( $p=0.99$ ) (Table 3). Levels of CRP were significantly higher in the MDR group, the mean value being $>3$ times higher than that of the non-MDR group $(p<0.001)$ (Table 3$)$. Leukocytosis was present in $80.0 \%$ of patients in Group 1 compared to $55.55 \%$ in Group 2 , but these values were not statistically significant. Significant differences were noted in the absolute number $(p=0.02)$ and the percentage of neutrophils $(p<0.001)$ between the 2 groups, with higher values in Group 1 .

\section{ROC Analysis and Performance Curves}

The ROC curves show good discriminative ability in differentiating groups for all of the 3: CRP $(\mathrm{AUC}=0.891$, 95\% confidence interval [CI]: 0.795-0.987), percentage of neutrophils (AUC $=0.76,95 \%$ CI: 0.605-0.916), and ANC 
Table 3. Laboratory findings for the patients in the 2 study groups (Group 1 - otitis media with MDR S. pneumoniae, Group 2 - otitis media with non-MDR S. pneumoniae)

\begin{tabular}{|c|c|c|c|}
\hline Variables & Group $1(n=15)$ & Group $2(n=27)$ & $p$ value \\
\hline Presence of nasopharyngeal carriage, $n(\%)$ & $6(40)$ & $12(44.44)$ & 0.99 \\
\hline \multicolumn{4}{|l|}{ CRP level, mg/L } \\
\hline Mean \pm SD & $72.23 \pm 62.92$ & $14.96 \pm 15.57$ & \multirow{2}{*}{$<0.001$} \\
\hline Range & $10.53-245.69$ & $0.24-47.37$ & \\
\hline \multicolumn{4}{|l|}{ Leukocytes, $n, \times 10^{3}$ cells $/ \mathrm{mm}^{3}$} \\
\hline Mean \pm SD & $15.71 \pm 4.74$ & $13.07 \pm 6.59$ & \multirow{2}{*}{0.06} \\
\hline Range & $7.05-24.31$ & $4.35-33.93$ & \\
\hline Presence of leukocytosis (present/absent) & $12 / 3$ & $15 / 12$ & 0.31 \\
\hline \multicolumn{4}{|l|}{ ANC, $n, \times 10^{3}$ cells $/ \mathrm{mm}^{3}$} \\
\hline Mean $\pm S D$ & $8.46 \pm 3.97$ & $5.22 \pm 4.5$ & \multirow{2}{*}{0.004} \\
\hline Range & $2.97-16.39$ & $1.32-23.41$ & \\
\hline \multicolumn{4}{|l|}{ Neutrophils, \% } \\
\hline Mean \pm SD & $52.85 \pm 13.49$ & $38.34 \pm 16.16$ & \multirow{2}{*}{0.004} \\
\hline Range & $26.4-67.7$ & $11.2-79.3$ & \\
\hline \multicolumn{4}{|l|}{ Thrombocytes, $n, \times 10^{3}$ cells $/ \mathrm{mm}^{3}$} \\
\hline Mean \pm SD & $433.2 \pm 229.01$ & $425.26 \pm 169.74$ & \multirow{2}{*}{0.56} \\
\hline Range & $238-1,203$ & $127-744$ & \\
\hline \multicolumn{4}{|l|}{ Thrombocyte count anomaly (present/absent) } \\
\hline Thrombocytosis & $3 / 12$ & $13 / 14$ & 0.12 \\
\hline Thrombocytopenia & $0 / 15$ & $1 / 26$ & 1 \\
\hline Presence of anemia & 8 & 11 & 0.52 \\
\hline
\end{tabular}

CRP, C-reactive protein; SD, standard deviation; ANC, absolute neutrophil count; S. pneumoniae, Streptococcus pneumoniae.

Fig. 3. ROC curves for CRP (continuous bold line), percentage of neutrophils (dashed line) and ANC (dotted line), CRP + percentage of neutrophils (dashed bold line), and CRP + ANC (dotted bold line). $\mathrm{ROC}$, receiver operating characteristic; CRP, C-reactive protein; ANC, absolute neutrophil count.

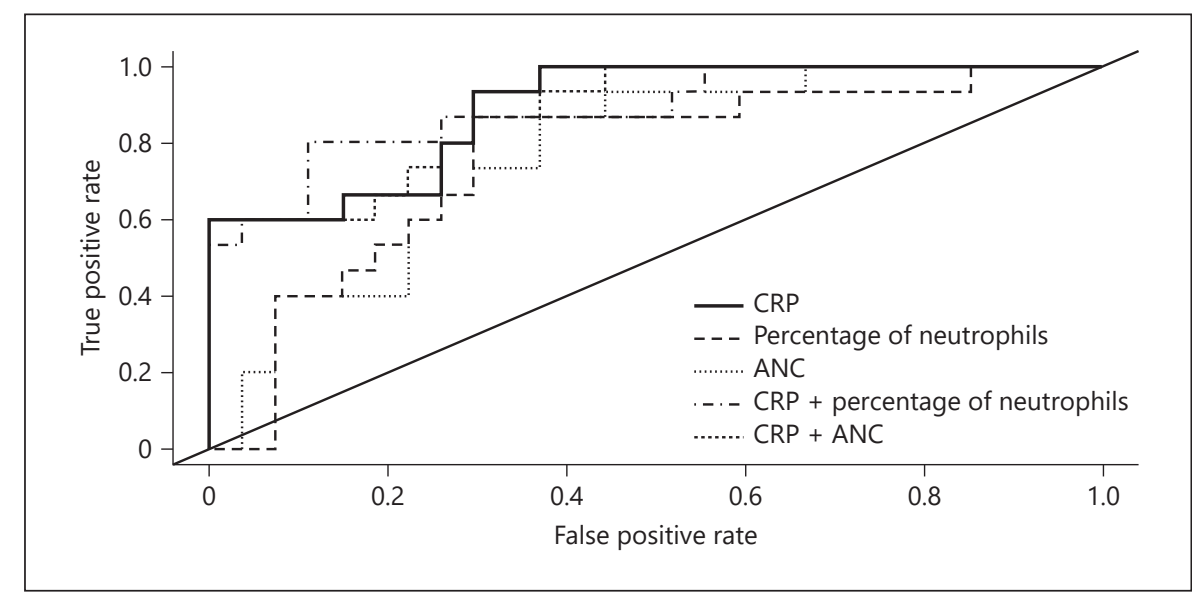

(AUC $=0.773,95 \%$ CI: 0.63-0.915) (Fig. 3). The cutoff value for CRP is $15.24 \mathrm{mg} / \mathrm{L}$, while the cutoff points for neutrophils and ANC were within normal range values (Table 4). The next step was to consider the combination of CRP with either ANC or percentage of neutrophils. In order to obtain the combined effect, a logistic regression was performed. The discriminative power of the 2 combinations was not superior to that of CRP alone (Fig. 3).

\section{Discussion}

The main finding in our study is that AOM cases with MDR S. pneumoniae were associated with a more severe inflammatory response than that of non-MDR strains, highlighted by higher values for CRP and ANC in the first $48 \mathrm{~h}$ of disease evolution. CRP values were most predictive for the presence of MDR in severe AOM with S. pneu- 
Table 4. Optimal cutoffs for CRP, neutrophils percentage, and ANC

\begin{tabular}{llll}
\hline $\begin{array}{l}\text { Variables (Youden/ } \\
\text { closest top-left) }\end{array}$ & Threshold & Sensitivity & Specificity \\
\hline CRP, mg \% & $15.24 / 15.24$ & $93.33 / 93.33$ & $70.37 / 70.37$ \\
Neutrophil percentage, \% & $39.9 / 39.9$ & $86.67 / 86.67$ & $70.37 / 70.37$ \\
ANC, $/ \mathrm{mm}^{3}$ & $4,888 / 4,888$ & $86.67 / 86.67$ & $62.96 / 62.96$
\end{tabular}

CRP, C-reactive protein; ANC, absolute neutrophil count.

moniae. Statistical analysis offered an optimal cutoff value of $15.24 \mathrm{mg} / \mathrm{L}$, with a sensitivity of $93 \%$ and a specificity of $70 \%$. However, for clinical practice, the purpose of a biomarker in such a situation is to identify cases at risk of evolving toward refractory AOM, avoiding at the same time unnecessary antibiotic treatment or tympanocentesis. In this setting, a higher specificity would be preferred. In our study group, the maximum CRP value for AOM patients with non-MDR S. pneumoniae was $47.37 \mathrm{mg} / \mathrm{L}$. If we are to use a cutoff CRP value of $50 \mathrm{mg} / \mathrm{L}$ to distinguish between the 2 groups of patients, a specificity of $100 \%$ would be reached, with a sensitivity of $60 \%$.

CRP is synthesized in the liver as a response to cytokines originating at the site of infection, particularly IL-6. Its rate of synthesis shadows the body's response to inflammation intensity. Its secretion begins within 4-6 h, doubling every $8 \mathrm{~h}$, and peaking at 36-50 h [16]. Due to this rapid increase in the first hours of the inflammation's onset, its values must be cautiously interpreted. This implies that the time at which a sample is taken for the CRP assay will be important, if it is to be used as a marker of MDR S. pneumoniae. In the present study, patient selection criteria required subjects with recent onset of AOM symptoms $(<48 \mathrm{~h})$ which falls within the CRP profile described above; furthermore, no previous antibiotic treatment was permitted. The rapid increase of CRP values in the first $48 \mathrm{~h}$ is also a possible explanation for the heterogeneous values in our study groups. Another possible cause is represented by the fact that the severity of AOM was established on clinical basis [7]. If body temperature is an objective parameter, pain remains subjective, its severity being sometimes difficult to assess in pediatric patients. For the duration of otorrhea, we found a significant statistical difference, lasting on average 2.4 days longer in the MDR group. A possible explanation is provided by the presence of mastoiditis and hence a more severe middle ear disease in the MDR group. An additional explanation is given by the time needed to obtain results of the MEF culture and therefore a delayed introduction of appropriate antibiotic treatment for the patients in the MDR group.

As in all pilot studies, it is important to recognize any limitations in the study design or imbalances in the patient population recruited. Differences between the inflammatory responses for the 2 groups can be partially explained by the different clinical manifestations of the $S$. pneumoniae infection. The majority of patients presented concurrent lower respiratory tract inflammation by associated viral or pneumococcal disease or complications. On the other hand, we must also consider that AOM is usually associated with concurrent upper respiratory tract infections [17]. In addition, the inflammatory response is influenced by the strain of $S$. pneumoniae that produces the infection. For example, S. pneumoniae 19A ST320 clone is known to induce a more severe inflammatory response as well as being predominantly MDR [4].

From a microbiological perspective, the mechanisms associated with resistance to antibiotics could also indicate an increase in virulence [18]. It has been suggested that this phenomenon could be partially explained by the localization of the genes encoding penicillin-binding proteins near the capsular locus in the pneumococcal genome [19], implying that resistance to $\beta$-lactams could be associated with certain serotypes with higher virulence. Moreover, resistance to different classes of antibiotics is sometimes associated between them. For example, TMPSMX resistance is frequently reported in S. pneumoniae isolates nonsusceptible to penicillin [20].

\section{Conclusions}

MDR in AOM with S. pneumoniae is associated with a more severe inflammatory response and a higher frequency of complications. Therefore, CRP, a commonly available and cheap laboratory test, may help the clinician to take into consideration the presence of an MDR strain. This pilot study provides the basis for a future larger study focusing on the differences between MDR versus nonMDR pneumococcus infection in AOM and to include patients who have received pneumococcal conjugate vaccine-immunization.

\section{Acknowledgements}

The authors thank Dr. Andrew Griffiths and Kate Chasmar for valuable scientific suggestions and language review. 


\section{Statement of Ethics}

This study was approved by the Ethics Committee of the Emergency Children's Hospital Louis Turcanu, Timisoara and complied with the Declaration of Helsinki (approval for publication no. 17816). Informed consent for the examination, diagnostic procedures, and the usage of the patients' data were agreed in writing by each patient's legal representative.

\section{Conflict of Interest Statement}

The authors have no conflicts of interest to declare.

\section{Author Contributions}

All authors contributed equally to this study. Raluca Horhat: otolaryngological examination and procedures, clinical input, literature review, and article writing. Raul Horhat: study design and data analysis. Valeria Mocanu: literature review and manuscript writing.

\section{Data Availability Statement}

The data that support the findings of this study are available in the „figshare" repository at https:// figshare.com/articles/software/ AOM/12959822.

\section{Funding Sources}

No external funding was obtained for this study.

\section{References}

1 Al-Mazrou KA, Shibl AM, Kandeil W, Pirçon JY, Marano C. A prospective, observational, epidemiological evaluation of the aetiology and antimicrobial susceptibility of acute otitis media in Saudi children younger than 5 years of age. J Epidemiol Glob Health. 2014;4(3): 231-8.

2 Loughran AJ, Orihuela CJ, Tuomanen EI. Streptococcus pneumoniae: invasion and inflammation. Microbiol Spectr. 2019;7(2).

3 Ngo CC, Massa HM, Thornton RB, Cripps AW. Predominant bacteria detected from the middle ear fluid of children experiencing otitis media: a systematic review. PLoS One. 2016;11(3):e0150949.

4 Chi H, Chiu NC, Huang FY, Hsu CH, Lee KS, Huang LM, et al. Acute otitis media caused by Streptococcus pneumoniae serotype 19A ST320 clone: epidemiological and clinical characteristics. J Microbiol Immunol Infect. 2018;51(3):337-43.

5 Ovnat Tamir S, Roth Y, Goldfarb A, Grotto I, Marom T. Severity of pneumococcal versus non-pneumococcal acute otitis media in children. Clin Otolaryngol. 2015;40(4):370-7.

6 Pichichero ME, Pichichero CL. Persistent acute otitis media: II. Antimicrobial treatment. Pediatr Infect Dis J. 1995;14(3):183-8.

7 Lieberthal AS, Carroll AE, Chonmaitree T, Ganiats T, Hoberman A, Jackson MA, et al. Clinical practice guideline. The diagnosis and management of acute otitis media. Pediatrics. 2013;131(3): e964-99. [published correction appears in: Pediatrics. 2014 Feb;133(2):346. Dosage error in article text].
8 Pitaro J, Waissbluth S, Quintal MC, Abela A, Lapointe A. Characteristics of children with refractory acute otitis media treated at the pediatric emergency department. Int J Pediatr Otorhinolaryngol. 2019;116:173-6.

9 Algammal AM, Enany ME, El-Tarabili RM, Ghobashy MOI, Helmy YA. Prevalence, antimicrobial resistance profiles, virulence and enterotoxins-determinant genes of MRSA isolated from subclinical bovine mastitis in Egypt. Pathogens. 2020;9:362.

10 Makharita RR, El-kholy I, Hetta HF, Abdelaziz MH, Hagagy FI, Ahmed AA, et al. Antibiogram and genetic characterization of carbapenem-resistant gram-negative pathogens incriminated in healthcare-associated infections. Infect Drug Resist. 2020;13:3991-4002.

11 Jorgensen JH, Barry AL, Traczewski MM, Sahm DF, McElmeel ML, Crawford SA. Rapid automated antimicrobial susceptibility testing of Streptococcus pneumoniae by use of the bioMerieux VITEK 2. J Clin Microbiol. 2000 Aug;38(8):2814-8.

12 Clinical and Laboratory Standards Institute. M100 performance standards for antimicrobial susceptibility testing. 27th ed. CLSI supplement. Wayne, PA: Clinical and Laboratory Standards Institute; 2017.

13 Eda S, Kaufmann J, Roos W, Pohl S. Development of a new microparticle-enhanced turbidimetric assay for C-reactive protein with superior features in analytical sensitivity and dynamic range. J Clin Lab Anal. 1998;12(3): $137-44$.
14 Kayadibi H, Acar IA, Cam S. Stability of complete blood count parameters depends on the storage temperature, storage time, transport position and selected stability criterion. Scand J Clin Lab Invest. 2020 Oct;80(6):470-8.

15 World Health Organization. Haemoglobin concentrations for the diagnosis of anaemia and assessment of severity. 2011. WHO reference number: WHO/NMH/NHD/ $\mathrm{MNM} / 11.1$.

16 R Core Team. R: a language and environment for statistical computing. Vienna, Austria: R Foundation for Statistical Computing; 2019. Available from: https://www.R-project.org/.

17 Van den Bruel A, Thompson MJ, Haj-Hassan T, Stevens R, Moll H, Lakhanpaul M, et al. Diagnostic value of laboratory tests in identifying serious infections in febrile children: systematic review. BMJ. 2011;342: 33082 .

18 Chonmaitree T, Revai K, Grady JJ, Clos A, Patel JA, Nair S, et al. Viral upper respiratory tract infection and otitis media complication in young children. Clin Infect Dis. 2008;46(6): $815-23$.

19 Algammal AM, Mabrok M, Sivaramasamy E, Youssef FM, Atwa MH, El-Kholy AW, et al. Emerging MDR-Pseudomonas aeruginosa in fish commonly harbor oprL and toxA virulence genes and blaTEM, blaCTX-M, and tetA antibiotic-resistance genes. Sci Rep. 2020;10:15961.

20 Reinert RR. The antimicrobial resistance profile of Streptococcus pneumoniae. Clin Microbiol Infect. 2009;15(Suppl 3):7-11. 